\title{
Rectal Ulcer
}

National Cancer Institute

\section{Source}

National Cancer Institute. Rectal Ulcer. NCI Thesaurus. Code C78590.

A circumscribed inflammatory and necrotic erosive lesion in the mucosa surface of the rectum. 